\title{
Gas density profile in dark matter halo in chameleon cosmology
}

\author{
Ayumu Terukina* and Kazuhiro Yamamotd \\ Department of Physical Science, Hiroshima University, Higashi-Hiroshima 739-8526, Japan
}

(Dated: March 24, 2022)

\begin{abstract}
We investigate the gas density, temperature, and pressure profiles in a dark matter halo under the influence of the chameleon force. We solve the hydrostatic equilibrium equation for the gas coupled with the chameleon field in an analytic manner, using an approximate solution for the chameleon field equation with the source term, with a generalized Navarro-Frenk-White universal density profile. We find that the gas distribution becomes compact because a larger pressure gradient is necessary due to the additional chameleon force. By confronting the theoretical prediction with the data of the temperature profile of the Hydra A cluster according to Suzaku x-ray observations out to the virial radius, we demonstrate that a useful constraint on a model parameter can be obtained depending on the value of the coupling constant. For example, the upper bound of the background value of chameleon field, $\phi_{\infty}<10^{-4} M_{\mathrm{Pl}}$, is obtained in the case $\beta=1$, where $\beta$ is the coupling constant between the chameleon field and the matter, and $M_{\mathrm{Pl}}$ is the Planck mass. However, the error of the present data is not so small that we obtain a useful constraint in the case $\beta=1 / \sqrt{6}$, which corresponds to an $f(R)$ model.
\end{abstract}

PACS numbers: 98.80.-k 98.65.Cw

The accelerated expansion of the Universe is one of the most fundamental mysteries in basic science. Longdistance modification of the gravity theory is a challenging approach to this problem. However, any gravity theory must pass the stringent constraints from the Solar System. The chameleon mechanism is a noble mechanism for screening a scalar degree of freedom which appears in a class of modified gravity models, depending on the density of matter in the local environment [1, 2]. Newtonian gravity is recovered in a high-density region, thereby evading the Solar System constraints. Recently, it has been pointed out that the modification of gravity might be detected using halos of galaxies and galaxy clusters, because the screening mechanism could not be complete in their outer regions [3] 8 ].

In the present paper, we focus on the gas distribution in a dark matter halo under the influence of the chameleon force. In Ref. 9], the authors found an analytic solution of the chameleon field, assuming the matter distribution of the Navarro-Frenk-White (NFW) universal density profile [10] (c.f. Ref. [11]). Utilizing their analytic method, we investigate the gas density, temperature, and pressure profiles under the influence of the chameleon force. We find that the chameleon force significantly influences the gas distribution. We also demonstrate a useful constraint on the chameleon gravity model from confronting the theoretical temperature profile with x-ray observations of a cluster of galaxies.

The chameleon field equation for a quasistatic system in the Einstein frame is

$$
\nabla^{2} \phi=V_{, \phi}+\frac{\beta}{M_{\mathrm{Pl}}} \rho e^{\beta \phi / M_{\mathrm{Pl}}},
$$

where $V$ is the potential, $\rho$ is the matter density, $\beta$ is the coupling constant, and we have defined the reduced Planck mass by $M_{\mathrm{Pl}}{ }^{2}=1 /(8 \pi G)$ with the gravitational constant $G$. Here, we assume $V(\phi)=\Lambda^{4+n} / \phi^{n}$, where $\Lambda$ is the mass dimension parameter and $n$ is the dimensionless parameter. We also assume $\beta \phi / M_{\mathrm{Pl}} \ll 1$. The coupling between the scalar field and the matter density is the key for the chameleon mechanism, as we see below.

We follow the analytic method in Ref. [9] to find a solution for Eq. (11). In the present paper, we assume the generalized NFW density profile $\rho(x)=\rho_{s} / x(1+x)^{b}$ with $x=r / r_{s}$, where $\rho_{s}$ and $r_{s}$ are the characteristic density and scale of a halo, respectively, and $b$ is a parameter. The NFW density profile is the case $b=2$. The mass within the radius $x$ of the halo is given by $M(x)=4 \pi r_{s}^{3} \int_{0}^{x} d x x^{2} \rho(x)$. Instead of the parameters $r_{s}$ and $\rho_{s}$, we introduce the virial mass $M_{\text {vir }}$ and the concentration parameter $c$, which are defined by $M_{\mathrm{vir}}=(4 \pi / 3) r_{\mathrm{vir}}{ }^{3} \Delta_{c} \bar{\rho}_{c}$ and $c=r_{\mathrm{vir}} / r_{s}$, where $\Delta_{c}$ is the ratio of the spherical overdensity $\bar{\rho}(<c)$ within the virial radius $r_{\text {vir }}$ to the critical density of the universe $\bar{\rho}_{c}$; i.e., $\Delta_{c}=\bar{\rho}(<c) / \bar{\rho}_{c}$, for which we adopt $\Delta_{c}=100$ in a spherical collapse model.

The analytic solution for Eq. (1) is obtained by matching the interior solution $\phi_{\text {int }}$ and the exterior solution $\phi_{\text {out }}$, where $\phi_{\text {int }}$ is given by solving Ep. (1) while neglecting the term on the left-hand-side, while $\phi_{\text {out }}$ is given by neglecting the first term on the right-hand-side of Eq. (1). Then, we find

$$
\phi(x)=\left\{\begin{array}{lr}
\phi_{s}\left[x(1+x)^{b}\right]^{1 /(n+1)} \equiv \phi_{\mathrm{int}}, & x<x_{c}, \\
-B \frac{1-(1+x)^{2-b}}{(b-2) x}-\frac{C}{x}+\phi_{\infty} \equiv \phi_{\mathrm{out}} & x>x_{c}
\end{array}\right.
$$

where we have defined $\phi_{s}=\left(n \Lambda^{n+4} M_{\mathrm{Pl}} / \beta \rho_{s}\right)^{1 /(n+1)}$, $B=\beta \rho_{s} r_{s}^{2} / M_{\mathrm{Pl}}$, and $C$ and $x_{c}$ are determined by solving 
the matching conditions at $x=x_{c}$ :

$$
\begin{aligned}
& C=B \frac{\left(1+x_{c}\right)^{2-b}-1}{b-2}+\phi_{\infty} x_{c} \\
& \quad-\phi_{s}\left[x_{c}\left(1+x_{c}\right)^{b}\right]^{1 /(n+1)} x_{c} \\
& \phi_{\infty}-B\left(1+x_{c}\right)^{1-b} \\
& =\phi_{s}\left(x_{c}\left(1+x_{c}\right)^{b}\right)^{1 /(n+1)}\left(1+\frac{(1+b) x_{c}+1}{(n+1)\left(1+x_{c}\right)}\right) .
\end{aligned}
$$

The validity of the analytic solution is demonstrated for the case $b=2$ in Refs. [5, 9].

Note that $\phi_{s}$ is the typical value of the chameleon field in the interior region, where the chameleon mechanism works. This means that $\phi_{s} \ll \phi_{\infty}$, because the chameleon mechanism screens the chameleon field. With this fact, Eqs. (3) and (44) are approximated as

$$
\begin{aligned}
& C \simeq B\left(\left(1+x_{c}\right)^{2-b}-1\right) /(b-2)+\phi_{\infty} x_{c}, \\
& \phi_{\infty}-B\left(1+x_{c}\right)^{1-b} \simeq 0,
\end{aligned}
$$

since we are considering the case $x_{c}=\mathcal{O}(1)$. Hence, the scalar field in the exterior region is independent of $n$ and $\Lambda$. This is important because the constraint we obtain becomes independent of $n$ and $\Lambda$.

In our modeling of the gas distribution in a dark matter halo, we make a few assumptions for simplicity. First, we assume that the dark matter dominates the dark halo described by the generalized NFW density profile. The halo density profile could be affected by the modification of the gravity theory; however, we assume the same profile, since its validity is partially supported by $N$-body simulations for the Dvali-Gabadadze-Porrati model and $f(R)$ model [3 [5, 12]. Recently, Ref. [5] confirmed this validity for an $f(R)$ model, and argued a qualitative explanation for the validity. Second, we assume that the baryon density is negligible in the dark matter halo, which allows us to neglect its effect on the scalar field equation. The effect of the baryon component is discussed in Ref. [13]. The validity of this assumption is also supported by the recent measurements of the density profile of a cluster halo through gravitational lensing, which show that the NFW profile fits the data well [14, 15]. Third, we assume that the scalar field is coupled with the baryon component as well as the dark matter component. For example, in an $f(R)$ model, the chameleon force is coupled with both the dark matter and the baryon components.

Within Newtonian gravity, a useful model of the gas density profile is considered in Refs. [13, 16]. By assuming hydrostatic equilibrium between the gas pressure and the gravitational force from the dark matter with the NFW density profile, the universal gas density, temperature and pressure profiles are derived. We follow the method of Refs. 13, 16], but taking into account the chameleon force as well as the gravitational force, we derive the gas distribution in a halo. Now the hydrostatic equilibrium gives

$$
\frac{(1+\epsilon)}{\rho_{g}} \frac{d P_{g}}{d r}=-\frac{1}{M_{\mathrm{Pl}}}\left[\frac{d \phi_{G}}{d r}+\beta \frac{d \phi}{d r}\right],
$$

where $\rho_{g}$ and $P_{g}$ are the gas density and the pressure, respectively, and $\epsilon=0$ unless explicitly stated otherwise. Here $\phi_{G}$ denotes the gravitational potential, given by solving the gravitational Poisson equation, $\triangle \phi_{G}=$ $\rho /\left(2 M_{\mathrm{Pl}}\right)$. For the generalized NFW density profile, we find the solution $\phi_{G}(x)=\phi_{0}\left[1-(1+x)^{2-b} /(b-2) x\right]$, where we define $\phi_{0}=-\rho_{s} r_{s}^{2} / 2 M_{\mathrm{Pl}}(b-1)$. We assume that the gas obeys the polytropic equation of state $P_{g} \propto \rho_{g} T_{g} \propto \rho_{g}^{\gamma}$ with the polytropic index $\gamma$ and the gas temperature $T_{g}$. Introducing the function $y_{g}(x)$ by $\rho_{g}(x)=\rho_{g}(0) y_{g}(x), P_{g}(x)=P_{g}(0) y_{g}^{\gamma}(x)$, and $T_{g}(x)=$ $T_{g}(0) y_{g}^{\gamma-1}(x)$, we obtain the solution

$$
\begin{aligned}
& y_{g}(x)= {\left[1-\frac{\mu m_{p}}{k T_{g}(0) M_{\mathrm{Pl}}(1+\epsilon)} \frac{\gamma-1}{\gamma}\right.} \\
&\left.\times\left(\phi_{G}(x)-\phi_{G}(0)+\beta \phi(x)-\beta \phi(0)\right)\right]^{1 /(\gamma-1)} \\
&= \begin{cases}{\left[1-A\left(1+\frac{(1+x)^{2-b}-1}{(b-2) x}\right.\right.} \\
\left.\left.-\frac{\beta \phi_{s}}{\phi_{0}}\left[x(1+x)^{b}\right]^{1 /(n+1)}\right)\right]^{1 /(\gamma-1)}, & \text { for } x<x_{c}, \\
{\left[1-A\left(1+\left(1+2 \beta^{2}\right) \frac{(1+x)^{2-b}-1}{(b-2) x}\right.\right.} & \text { for } x>x_{c}, \\
\left.\left.-\frac{\beta}{\phi_{0}}\left(\phi_{\infty}-\frac{C}{x}\right)\right)\right]^{1 /(\gamma-1)},\end{cases}
\end{aligned}
$$

where we have defined $A=-\mu m_{p} \phi_{0}(\gamma-$ 1) $/ k T_{g}(0) M_{\mathrm{Pl}}(1+\epsilon) \gamma, \quad k$ is the Boltzmann constant, and $\mu m_{p}$ represents the mean molecular mass. We determine the parameter $\gamma$ by Expression (17) in Ref. [17]. Our conclusions are not altered qualitatively for the assumption on $\gamma$ within $1.1 \leq \gamma \leq 1.3$.

Figure 1 shows the gas density profiles, comparing the case with the chameleon force (solid curves) and the case of Newtonian gravity (dashed curves), adopting virial masses of $M_{\text {vir }}=10^{13} M_{\odot}, 10^{14} M_{\odot}$, and $4 \times 10^{14} M_{\odot}$, from top to bottom, respectively. The gas density decreases rapidly in the outer region (see the solid curves), where the chameleon force is influential. For the large mass cluster, the chameleon mechanism works out to large radii, because the density of dark matter is high enough even outside the halo. On the other hand, for the small mass cluster, the chameleon mechanism works only at small radii, because the dark matter density is high only in the central region. Because the chameleon force is an attractive force, a larger pressure gradient is necessary for balancing between them. This makes the gas distribution compact. This feature is more significant for the smaller-mass halo. 


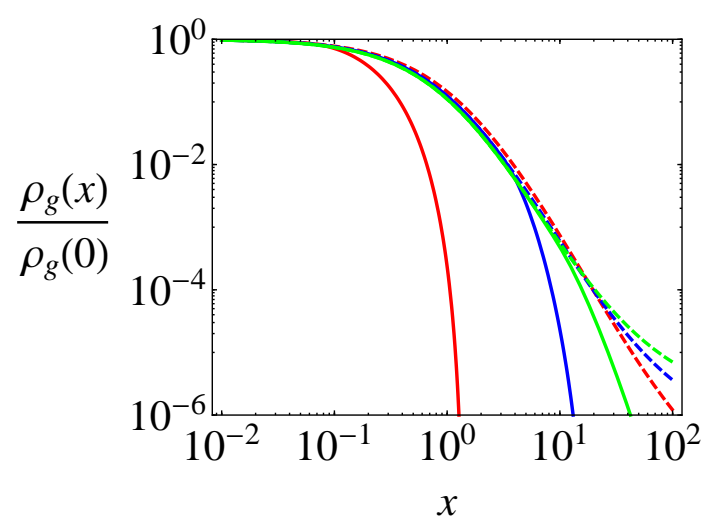

FIG. 1: Gas density profile as a function of the radius $r / r_{s}$. The solid (dashed) curves are with (without) the chameleon force, with virial masses $M_{\text {vir }}=4 \times 10^{14} M_{\odot}$ (green light curve), $10^{14} M_{\odot}$ (blue dark curve), and $M_{\mathrm{vir}}=10^{13} M_{\odot} \quad($ red curve), from top to bottom, respectively. Here we have adopted $\beta=1, n=1.8 \times 10^{-5}, \Lambda=2.4 \times 10^{-3} \mathrm{eV}$, and $\phi_{\infty}=1.1 \times 10^{-5} M_{\mathrm{P} 1}$.

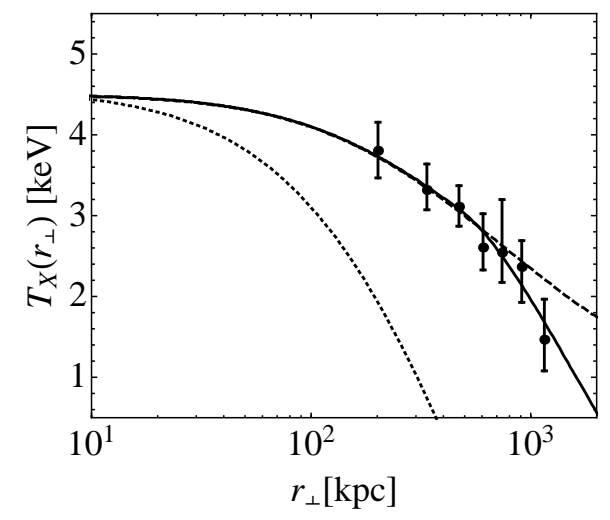

FIG. 2: Temperature profiles as a function of the radius $r_{\perp}$. The points with error bars show the observation data of the filament direction [18]. The curves show our theoretical model. The solid curve adopts the bestfit parameters $\left(\phi_{\infty}, M_{\mathrm{vir}}, c, T_{g}(0)\right)=\left(5.4 \times 10^{-5} M_{\mathrm{Pl}}, 5.1 \times\right.$ $\left.10^{14} M_{\odot}, 5.8,4.9 \mathrm{keV}\right)$. The dashed curve adopts $\phi_{\infty}=2 \times$ $10^{-5} M_{\mathrm{Pl}}$, while the dotted curve adopts $\phi_{\infty}=1.3 \times 10^{-4} M_{\mathrm{Pl}}$, where the other parameters are the same as those of the solid curve. Here we have fixed $\beta=1$ and $b=2$.

Using this characteristic feature, let us consider a constraint on the chameleon gravity model. To this end, we consider x-ray observations of a cluster of galaxies. Because of the steep drop of the gas density in the presence of the chameleon force, a similar drop in the x-ray surface brightness may appear in the outer region. In the present paper, we compare the x-ray temperature profile from the data reported from Suzaku observations of the Hydra A cluster out to the virial radius 18]. The Hydra A cluster is a medium-sized cluster located at a distance of $230 \mathrm{Mpc}$. Two different fields are observed

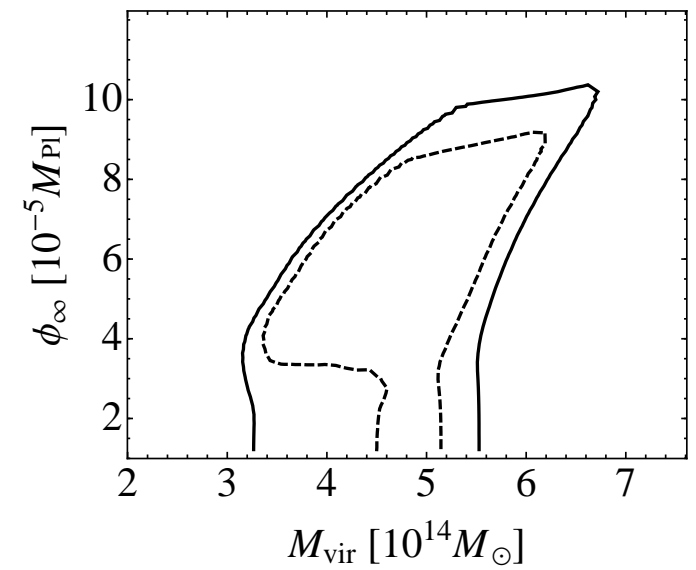

FIG. 3: The contours of $\Delta \chi^{2}$ on the parameter plane $\phi_{\infty^{-}}$ $M_{\text {vir }}$. Here we have fixed $\beta=1$ and $b=2$, but $c$ and $T_{g}(0)$ are varied as fitting parameters. The contour levels of the inner dashed curve and the outer solid curve are $\Delta \chi^{2}=2.7$ and 6.6 , respectively.

in Ref. [18]. One is the northwest offset from the x-ray peak of the cluster, and the other is the northeast offset. The former and latter fields are called the filament and void, respectively, because each field continues into the filament and void structures. In Fig. 2, the points with error bars show the data of the filament direction in Ref. [18].

The curves in Fig. 2 show our theoretical model of the x-ray surface brightness temperature, computed with the formula

$$
T_{X}\left(r_{\perp}\right)=\frac{\int \lambda_{c}\left(T_{g}\right) \rho_{g}^{2}\left(\sqrt{r_{\perp}^{2}+z^{2}}\right) T_{g}\left(\sqrt{r_{\perp}^{2}+z^{2}}\right) d z}{\int \lambda_{c}\left(T_{g}\right) \rho_{g}^{2}\left(\sqrt{r_{\perp}^{2}+z^{2}}\right) d z}
$$

where $\lambda_{c}\left(T_{g}\right)$ is the cooling function, for which we have assumed $\lambda_{c}\left(T_{g}\right) \propto T_{g}^{1 / 2}$ (e.g., Ref. [19]), and $r_{\perp}$ is the radial coordinate perpendicular to the line-of-sight direction. The solid curve is the best-fit curve, whose parameters are noted in the caption. The dashed curve and the dotted curve adopt $\phi_{\infty}=2 \times 10^{-5} M_{\mathrm{Pl}}$ and $1.3 \times 10^{-4} M_{\mathrm{Pl}}$, respectively, where the other parameters are the same as those for the solid curve.

The dotted curve, the solid curve, and the dashed curve in Fig. 22represent the characteristic curves which appear when we vary $\phi_{\infty}$ from a sufficiently large value to a smaller one. First, the dotted curve represents the limit of the modified gravity. Namely, for the large value of $\phi_{\infty} \geq B, x_{c}$ becomes negative from Eq. (6). This means that there appears no interior region in a halo where the chameleon mechanism works to recover Newtonian gravity. Thus, for the case $\phi_{\infty} \geq B$, we have $\phi(x)=\phi_{\text {out }}(x)$ for the entire region, and therefore the solution Eq. (8) 
should be replaced with

$$
y_{g}(x)=\left[1-A\left(1+2 \beta^{2}\right)\left(1+\frac{(1+x)^{2-b}-1}{(b-2) x}\right)\right]^{1 /(\gamma-1)} .
$$

On the other hand, the dashed curve is the same as the limit of Newtonian gravity. For a small value of $\phi_{\infty}$, we have a large value of $x_{c}$ from Eq. (6). This means that the chameleon force is influential only at very large radii. Note that the interior solution $y_{g}(x)$, Eq. (9) for $x<x_{c}$, can be approximated by taking the limit of $\beta \rightarrow 0$ in Eq. (11), because $\phi_{s}$ takes a very small value to screen the scalar field where the chameleon mechanism works. In summary, the dotted curve and the dashed curve are the two opposite limits, and our theoretical curve is restricted by these two limits. Note that the limit of the modified gravity Eq. (11) depends on the coupling constant $\beta$.

Figure 3 shows the contours of $\Delta \chi^{2}$ on the parameter plane for $\phi_{\infty}$ and $M_{\mathrm{vir}}$, where $\chi^{2}$ is simply defined by $\chi^{2}=\sum_{i=1}^{7}\left(T_{X}\left(r_{\perp, i}\right)-T_{i}^{\text {obs. }}\right)^{2} /\left(\Delta T_{i}^{\text {obs. }}\right)^{2}$, where $T_{i}^{\text {obs. }}$ and $\Delta T_{i}^{\text {obs. }}$ are the observed data and the error of the filament direction, respectively, and $T_{X}\left(r_{\perp, i}\right)$ is our theoretical model. Here, we have fixed $\beta=1$ and $b=2$, but the parameters $c$ and $T_{g}(0)$ are varied so as to minimize $\chi^{2}$ within the range $3 \leq c \leq 10$ and $T_{0}^{*} / \alpha \leq T_{g}(0) \leq T_{0}^{*} \alpha$ with $\alpha=1.1$, where $T_{0}^{*}$ is given by Eq. (19) in Ref. [17]. When taking $T_{g}(0)$ as a completely free parameter, it is difficult to obtain a useful constraint from the present data due to the degeneracy between $T_{g}(0)$ and $M_{\text {vir }}$. The minimum value of $\chi^{2}$ is 1.0, where the number of degrees of freedom is 3 . The behavior of the contour is explained by the fact that the theoretical curve approaches that of Newtonian gravity as $\phi_{\infty}$ becomes small and that the steep drop becomes significant as $\phi_{\infty}$ increases. Figure 3 gives an upper bound of $\phi_{\infty}<10^{-4} M_{\mathrm{Pl}}$ at the 2-sigma level for the case $b=2$ and $\beta=1$. We obtain a similar upper bound of $\phi_{\infty}$ for different values of $b$, which are summarized in Table \ The upper bound of $\phi_{\infty}$ becomes larger for smaller $b$, but we may conclude that the results do not significantly depend on $b$. Table \includes the results with the void direction. The upper bound of $\phi_{\infty}$ depends on the data, i.e., the filament direction and the void direction; however, our conclusion does not alter significantly.

\begin{tabular}{c|c|c}
\hline \multirow{2}{*}{} & \multicolumn{2}{|c}{ Upper limit for $\phi_{\infty}$ in unit of $\left[M_{\mathrm{Pl}}\right]$} \\
\cline { 2 - 3 } & Filament & Void \\
\hline$b=1.7$ & $1.4 \times 10^{-4}$ & $0.9 \times 10^{-4}$ \\
\hline$b=2.0$ & $1.0 \times 10^{-4}$ & $0.8 \times 10^{-4}$ \\
\hline$b=2.5$ & $0.8 \times 10^{-4}$ & $0.6 \times 10^{-4}$ \\
\hline
\end{tabular}

TABLE I: Upper bounds of $\phi_{\infty}$ at the 2-sigma level for different values of $b$ and the data for the filament and void directions. Here we have fixed $\beta=1$.
So far, we have considered the case $\beta=1$; let us now discuss the case $\beta=1 / \sqrt{6}$, which corresponds to an $f(R)$ model [20]. In this case, we could not obtain a useful constraint on $\phi_{\infty}$, which is explained as follows. The theoretical density profile is limited by two characteristic curves, Eq. (11) and Eq. (11) with $\beta=0$. When $\beta$ is small, the difference between these two characteristic curves is small, because the drop of the gas density is not steep. This is the reason why no useful constraint on the $f(R)$ model was obtained from the present x-ray data here.

In summary, under the assumption of hydrostatic equilibrium between the gas pressure gradient and the gravitational and chameleon forces, we derived the gas density profile in an analytic manner. Here we assumed the polytropic equation of state for the gas and the generalized NFW density profile for the dark matter distribution. The chameleon force may give rise to a steep drop in the gas distribution in the outer region of a halo. This feature is more significant when the mass of a halo is small and $\beta$ and $\phi_{\infty}$ are large. The gas density profile depends on $\beta$ and $\phi_{\infty}$, but it does not depend on $n$ and $\Lambda$. This provides us with an opportunity to constrain $\beta$ and $\phi_{\infty}$ by comparison with observations. We demonstrated a constraint on $\phi_{\infty}$ in the chameleon gravity model, using the data of the temperature profile from the x-ray observation [18]. We obtained a useful upper bound of $\phi_{\infty}<10^{-4} M_{\mathrm{Pl}}$ in the case $\beta=1$ [21]; however, no useful constraint was obtained in the case $\beta=1 / \sqrt{6}$, which corresponds to an $f(R)$ model. In order to obtain a useful constraint, observations of the outer region of a smaller mass cluster are more advantageous. Furthermore, a combination with other observations like the weak lensing measurements might improve the constraint. In our investigation, the assumption of the hydrostatic equilibrium of hot gas might be crucial. To estimate the effect of deviation from it, we obtained similar constraints by adopting the nonzero values of $\epsilon= \pm 0.5$ in Eq. (77). The upper bound of $\phi_{\infty}$ changes from $10^{-4} M_{\mathrm{Pl}}$ for $\epsilon=0$ to $0.6 \times 10^{-4} M_{\mathrm{Pl}}$ and $2.1 \times 10^{-4} M_{\mathrm{Pl}}$ for $\epsilon=0.5$ and -0.5 , respectively. Thus, the assumption of hydrostatic equilibrium is crucial to the constraint, but we may obtain a useful constraint if we can model the state of the gas correctly. Further study is necessary for this problem. Finally, we assumed spherical symmetry for a halo, an assumption whose validity must be checked when comparing with observational data. In the present paper, the results in Table \ do not depend on the filament direction and the void direction significantly, which suggests the validity of our assumption.

Acknowledgments We thank R. Kimura and T. Narikawa for fruitful discussions. We also thank S. Nishino, Y. Fukazawa, T. Kobayashi, K. Koyama, R. C. Nichol, A. Taruya, and Y. Suto for useful conversation related to the topic in the present paper. This work was supported by a Grant-in-Aid for Scientific Re- 
search from the Japanese Ministry of Education, Culture, Sports, Science, and Technology (Grants No. 21540270, No. 21244033).

* Email telkina" at" theo.phys.sci.hiroshima-u.ac.jp

† Email kazuhiro" at" hiroshima-u.ac.jp

[1] J. Khoury and A. Weltman, Phys. Rev. D 69, 044026 (2004).

[2] D.F. Mota and J.D. Barrow, Phys. Lett. B 581, 141 (2004).

[3] F. Schmidt, Phys. Rev. D 81, 103002 (2010).

[4] T.Y. Lam, T. Nishimichi, F. Schmidt, and M. Takada, et al., Phys. Rev. Lett. 109, 051301 (2012).

[5] L. Lombriser, K. Koyama, G.-B. Zhao, and B. Li, Phys. Rev. D 85, 124054 (2012).

[6] L. Lombriser, F. Schmidt, T. Baldauf, R. Mandelbaum, U. Seljak, and R.E. Smith, Phys. Rev. D 85, 102001 (2012).

[7] T. Narikawa, R. Kimura, T. Yano, and K. Yamamoto, Int. J. Mod. Phys. D 20, 2383 (2011).

[8] T. Narikawa and K. Yamamoto, J. Cosmol. Astropart. Phys. 05 (2012) 016.

[9] R. Pourhasan, N. Afshordi, R.B. mann, and A.C. Davis, J. Cosmol. Astropart. Phys. 12 (2011) 005.
[10] J.F. Navarro, C.S. Frenk and S.D.M. White, Astrophys. J. 490, 493 (1997).

[11] P. Burikham and S. Panpanichi, Int. J. Mod. Phys. D 21, 1250041 (2012).

[12] G.-B. Zhao, B. Li and K. Koyama, Phys. Rev. Lett. 107, 071303 (2011).

[13] Y. Suto, S. Sasaki, and N. Makino, Astrophys. J. 509, 544 (1998).

[14] K. Umetsu, T. Broadhurst, A. Zitrin, E. Medezinski, D. Coe, and M. Postman, Astrophys. J. 738, 41 (2011).

[15] M. Oguri, M.B. Bayliss, H. Dahle, K. Sharon, M.D. Gladders, P. Natarajan, J.F. Hennawi, and B.P. Koester, Mon. Not. R. Astron. Soc. 420, 3213 (2012).

[16] E. Komatsu and U. Seljak, Mon. Not. R. Astron. Soc. 327, 1353 (2001).

[17] E. Komatsu and U. Seljak, Mon. Not. R. Astron. Soc. 336, 1256 (2002).

[18] T. Sato et al., arXiv:1203.1700 [Publ. Astron. Soc. Jpn. (to be published)].

[19] V.R. Eke, J.J. Navarro, and C.S. Frenk, Astrophys. J. 503, 569 (1998)

[20] A. A. Starobinsky, JETP Lett. 86, 157 (2007); W. Hu and I. Sawicki, Phys. Rev. D 76, 064004 (2007); S. Tsujikawa, PRD 77, 023507 (2008).

[21] When we take the environment effect into account, this bound might be understood as the bound around the Hydra A cluster. 